\title{
Simulation numérique du soudage de grandes structures par une approche locale/globale
}

\author{
YongGang DuAn $^{1, a}$, FrédÉRIC Boitout ${ }^{2}$, JEAN-BAPtiste LEBlond $^{3}$ \\ ET JeAN-Michel BergheaU ${ }^{1}$ \\ 1 Laboratoire de Tribologie et Dynamique des Systèmes, UMR 5513 CNRS/ECL/ENISE, \\ École Nationale d'Ingénieurs de Saint-Étienne, 58 rue Jean Parot, 42023 Saint-Étienne Cedex 2, France \\ 2 ESI Group, Le Récamier, 70 rue Robert, 69458 Lyon Cedex 06, France \\ 3 LMM UMR 7607 CNRS/Université Paris VI, Tour 65-55, 4 place Jussieu, 75252 Paris Cedex 05, France
}

Reçu le 25 mai 2007, accepté le 24 janvier 2008

\begin{abstract}
Résumé - Le soudage est une des techniques d'assemblage les plus utilisées dans les secteurs de l'aéronautique, de l'automobile et de la construction navale. Un des principaux enjeux industriels est de limiter l'utilisation des outils de bridage de façon à réduire le coût et faciliter l'automatisation des lignes d'assemblage. Une façon de répondre à cette demande est de chercher à optimiser le procédé de soudage et la séquence de soudage. Cependant, l'optimisation expérimentale nécessite la mise en place de prototypes et de mesures coûteuses qui limitent le nombre de solutions pouvant être étudiées. La simulation par éléments-finis peut être utilisée à cette fin, cependant elle est rendue difficile car, d'une part, elle met en jeu des phénomènes physiques complexes et, d'autre part, alors que des modèles locaux de la soudure sont généralement suffisants pour prédire les contraintes résiduelles, des modèles globaux sont nécessaires pour évaluer les distorsions. Une approche locale/globale [1] a ainsi été développée pour déterminer les distorsions résiduelles de soudage des structures de taille importante. Cette méthode consiste à calculer les déformations plastiques sur un modèle local et à les projeter ensuite sur un modèle global. Les distorsions dues au soudage sont alors obtenues par une simple analyse élastique. Dans cet article, le principe de l'approche locale/globale est tout d'abord présenté. On s'intéresse ensuite à la définition du modèle local en terme de taille et de conditions aux limites à lui appliquer.
\end{abstract}

Mots clés : Soudage / distorsions résiduelles / approche locale/globale / grandes structures / simulation numérique

\begin{abstract}
Numerical simulation of welding of large structures using a local/global approach. Welding technology is one of the main joining techniques used in industry for parts assembly in aeronautics, automobiles and ships. One major industrial concern is to limit the use of clamping tools to reduce the cost and facilitate the automation of assembly lines. Optimization of the welding sequence and process is one way to reach this goal. However, experimental optimization requires prototyping and measurements which are extremely expensive and time consuming and finally, very few solutions can be used. Finite-element simulations can be used in that aim but the difficulty is, on one hand, that welding processes involve complex physical phenomena and, on the other hand, that where local models are sufficient to predict stresses, only global 3D models can correctly evaluate distortions. A local/global approach [1] has thus been developed to determine the welding residual distortions of large structures. This method consists in calculating the plastic strains on a local model and projecting them to a global model. The distortions due to welding are then obtained through a simple elastic analysis. In this article, the principle of the local/global approach is presented firstly. The definition of the local model is studied then in terms of the size of model and applied boundary conditions.
\end{abstract}

Key words: Welding / residual distortions / local / global approach / large structures / numerical simulation

a Auteur pour correspondance : yong-gang.duan@enise.fr 


\section{Nomenclature}

\begin{tabular}{|ll|}
\hline$C$ & Chaleur spécifique $\left(\mathrm{J} \cdot \mathrm{kg}^{-1} \cdot \mathrm{K}^{-1}\right)$ \\
$E$ & Module de Young (MPa) \\
$H_{\mathrm{c}}$ & Coefficient de convection $\left(\mathrm{W} \cdot \mathrm{m}^{-2} \cdot \mathrm{K}^{-1}\right)$ \\
$n$ & Normale unitaire \\
$t$ & Temps (s) \\
$T$ & Température $(\mathrm{K})$ \\
$T_{0}$ & Température du milieu extérieur $(\mathrm{K})$ \\
$\epsilon$ & Émissivité de la surface supposée $\ll$ grise $»(\epsilon=0,8)$ \\
$\lambda$ & Conductivité thermique $\left(\mathrm{W} \cdot \mathrm{m}^{-1} \cdot \mathrm{K}^{-1}\right)$ \\
$\sigma$ & Constante de Stefan $\left(\sigma=5,67 \times 10^{-8} \mathrm{~W} \cdot \mathrm{m}^{-2} \cdot \mathrm{K}^{-4}\right)$ \\
\hline
\end{tabular}

\section{Introduction}

Le soudage est une des techniques d'assemblage les plus utilisées dans les secteurs de l'aéronautique, de l'automobile et de la construction navale. Un des principaux enjeux industriels est de limiter l'utilisation des outils de bridage de façon à réduire le coût et faciliter l'automatisation des lignes d'assemblage. Une façon de répondre à cette demande est de chercher à optimiser le procédé de soudage et la séquence de soudage. Cependant, l'optimisisation expérimentale nécessite la mise en place de prototypes et de mesures coûteuses qui limitent le nombre de solutions pouvant être étudiées.

La simulation par éléments-finis peut être utilisée à cette fin, cependant elle est rendue difficile car, d'une part, elle met en jeu des phénomènes physiques complexes et, d'autre part, alors que des modèles locaux de la soudure sont généralement suffisants pour prédire les contraintes résiduelles, des modèles globaux tridimensionnels sont nécessaires pour évaluer les distorsions. Compte tenu des importants gradients de température, phases, contraintes et déformations plastiques qu'il existe dans les Zones Affectées Thermiquement (ZAT) de soudage, des maillages extrêmement fins sont nécessaires pouvant ainsi conduire à des tailles de modèles gigantesques et à des temps de calcul prohibitifs. De nombreuses méthodes ont donc été proposées pour rendre possibles de tels calculs [2-6].

Mourgue et al. [1,7] ont développé une approche locale/globale pour déterminer les distorsions résiduelles des structures minces de grandes dimensions. Cette méthode consiste à ne simuler qu'une petite partie du cordon de soudure en utilisant un maillage local, et à projeter les déformations plastiques, en tant que « déformations initiales », sur un maillage coque de la structure complète. Les distorsions résiduelles peuvent alors être déterminées par une simple analyse élastique.

Dans cet article, le principe de l'approche locale/globale est présenté. On s'intéresse ensuite à la définition du modèle local en terme de taille et de conditions aux limites à lui appliquer.

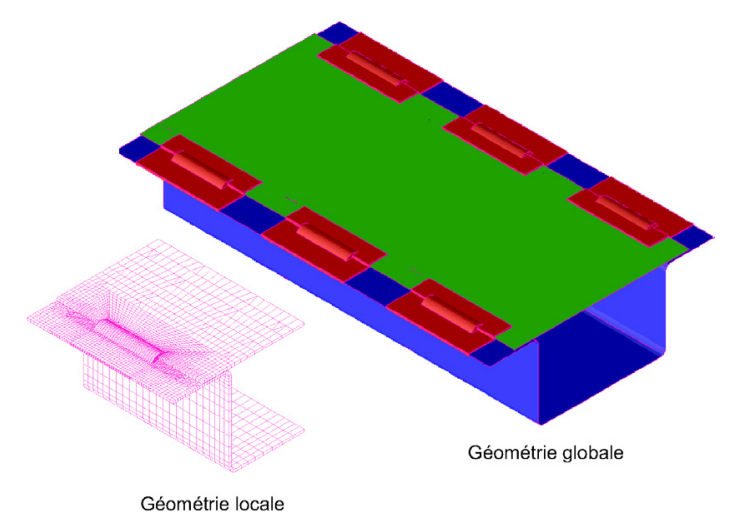

Fig. 1. Modèles local et global.

\section{Principe de l'approche locale/globale}

L'approche locale/globale proposée par Mourgue et al. [1] s'appuie sur deux niveaux de modélisation et vise à coupler les résultats obtenus sur un modèle local (premier niveau) avec une simulation globale sur l'ensemble de la structure (deuxième niveau) (Fig. 1).

Les déformations plastiques et la microstructure métallurgique induites par le soudage sont supposées être localisées à proximité du joint soudé. Il est donc possible de les déterminer en utilisant un modèle 3D local, ne concernant que le joint et son voisinage. Au niveau de la structure complète, les distorsions sont seulement dues aux déformations plastiques locales induites par le soudage et le comportement global de la structure peut être supposé élastique. Les déformations plastiques locales sont donc reportées, en tant que déformations initiales, dans un modèle 3D global, concernant cette fois toute la structure et évidemment beaucoup moins fin que le modèle local. Une simple analyse élastique peut alors être réalisée pour déterminer les distorsions résiduelles.

Une possibilité pour ce faire est d'utiliser un macroélément tel que proposé par Souloumiac et al. [7] pour intégrer la soudure dans un maillage en éléments-finis de coque.

Mais, la simulation locale doit refléter les conditions de soudage telles qu'elles existent sur la structure réelle. Il s'agit tout d'abord de retrouver des distributions de températures correctes sur un modèle incomplet de la structure et ensuite, de reproduire correctement les conditions mécaniques imposées par le reste de la structure.

\section{3. Étude de la définition du modèle local}

\subsection{Propositions}

Pour les aspects thermiques, une première idée consiste à réaliser des simulations simples $3 \mathrm{D}$ à l'aide de la technique de «transformation de l'espace »sur un modèle de structure simplifiée de type plaque pour laquelle le maillage serait étiré afin de reproduire la diffusion de la chaleur. Cela permet de déterminer des dimensions raisonnables pour le modèle local. On pourra 


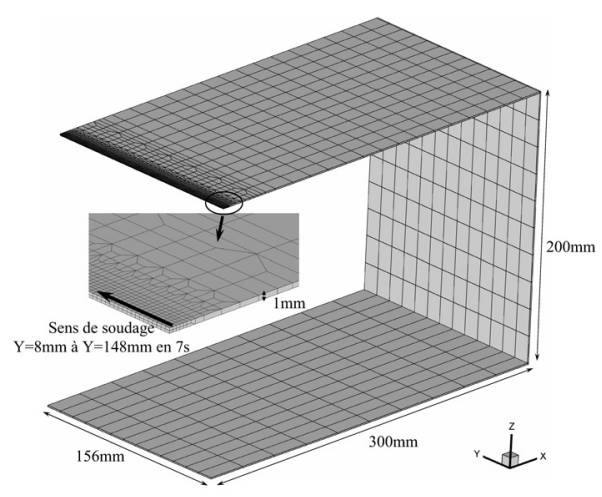

Fig. 2. Modèle de référence.

en effet choisir des dimensions de façon à ce que la température soit à peu près homogène dans l'épaisseur sur les frontières. Une telle approche nous laisse cependant une certaine latitude sur les dimensions qui peuvent être plus ou moins importantes tout en respectant le critère précédemment énoncé. Le choix des dimensions optimales dépendra alors des considérations mécaniques. Une fois cette taille déterminée, il sera alors possible de réaliser des calculs par éléments-finis thermo-métallurgiques couplés sur un modèle local prolongé.

En ce qui concerne la partie mécanique, l'influence des dimensions du modèle local et des conditions aux limites appliquées à sa frontière sur les déformations plastiques calculées doit être étudiée. Certains types de conditions aux limites sont donc testés ici. Une façon de statuer sur les dimensions optimales du modèle local est de tracer l'écart moyen par rapport à un modèle de référence. Cela permet à l'utilisateur de choisir son modèle en fonction du coût qu'il souhaitera y mettre.

\subsection{Modèle de référence}

On considère le cas de soudage de la figure 2. La source de chaleur se déplace au milieu de la face supérieure pour laisser une soudure de $140 \mathrm{~mm}$ de long. Le matériau est de l'acier 16MND5 choisi pour ses caractéristiques connues [8].

Le procédé de soudage de cette structure étant symétrique, seule la moitié de la structure est modélisée. Le maillage comporte 19834 nœuds pour 6799 éléments du second ordre (3666 éléments de peau, 3133 éléments 3D). Les éléments sont assez grossiers le long de la soudure où ils mesurent $0,8 \times 1 \times 0,5 \mathrm{~mm}$ (Fig. 2$)$. La dimension des éléments les plus éloignés de la soudure est de $30 \times 12 \times 1 \mathrm{~mm}$.

La source de chaleur appliquée sur la face supérieure est une source surfacique de forme gaussienne de rayon $2,5 \mathrm{~mm}$ dont l'intensité maximale est de $35 \mathrm{~W} . \mathrm{mm}^{-2}$. Cette source se déplace à une vitesse de $20 \mathrm{~mm} . \mathrm{s}^{-1}$. Les pertes par convection et rayonnement sont prises en compte avec :

$$
\lambda \cdot \operatorname{grad}(T) \cdot n=H_{\mathrm{c}}\left(T_{0}-T\right)+\sigma \epsilon\left(T_{0}^{4}-T^{4}\right)
$$

où $\lambda$ est la conductivité thermique, $T$ représente la température absolue, $n$ la normale unitaire, $H_{\mathrm{c}}$ le coefficient de convection $\left(H_{\mathrm{c}}=25 \mathrm{~W} \cdot \mathrm{m}^{-2} \cdot \mathrm{K}^{-1}\right), \sigma$ est la constante de Stefan $\left(\sigma=5,67 \times 10^{-8} \mathrm{~W} \cdot \mathrm{m}^{-2} \cdot \mathrm{K}^{-4}\right)$, $\epsilon$ est l'émissivité de la surface $(\epsilon=0,8)$, et $T_{0}$ est la température du milieu extérieur $\left(T_{0}=20{ }^{\circ} \mathrm{C}\right)$. Ces valeurs sont artificiellement augmentées d'un facteur 1,5 sur la face inférieure pour tenir compte des pertes supplémentaires dues au support.

$\mathrm{Du}$ point de vue métallurgique, 4 phases sont considérées : austénite, ferrite-perlite, bainite, martensite. Toutes les propriétés thermiques et mécaniques, à l'exception du coefficient de Poisson, sont considérées dépendantes de la température et/ou des phases. La plasticité de transformation est prise en compte et le matériau est supposé avoir un écrouissage de type isotrope. La simulation mécanique est réalisée dans l'hypothèse des petites perturbations. Les déformations plastiques ne sont présentées que dans le voisinage de la soudure, la taille de la zone affectée par ces déformations étant de l'ordre de $4 \mathrm{~mm}$.

\section{3. Étude thermique}

La méthode de «transformation de l'espace » a été introduite dans SYSTUS ${ }^{\mathrm{TM}}[9]$ pour traiter les problèmes définis sur des domaines non bornés. Afin de reproduire la diffusion de la chaleur dans les zones lointaines et de déterminer des dimensions raisonnables pour le modèle local, une simulation est effectuée à l'aide de cette méthode.

Pour ce type de source de chaleur peu pénétrante, les distributions de température sont à peu près homogènes dans l'épaisseur, et ce à partir d'une distance d'environ $5 \mathrm{~mm}$ de la source. Il est donc raisonnable de choisir cette valeur comme largeur minimum du maillage local. C'est elle qui sera utilisée pour la simulation mécanique.

\subsection{Dimensions optimales du modèle local}

Les modèles locaux prolongés avec des éléments coques sont utilisés pour les calculs thermométallurgiques couplés (Fig. 3a), en tenant compte de la largeur minimum précédemment définie. Ainsi la largeur de la partie 3D est prise supérieure ou égale à $5 \mathrm{~mm}$. La source de chaleur appliquée et le matériau sont les mêmes que lors du cas précédent. Le modèle concernant le calcul mécanique est composé seulement d'éléments 3D (Fig. 3b) et une partie, de largeur $4 \mathrm{~mm}$, est choisie pour effectuer la comparaison avec le modèle de référence.

\subsubsection{Conditions aux limites « frontière rigide »}

Pour tester l'influence des dimensions du modèle local et des conditions aux limites appliquées à sa frontière sur les déformations plastiques calculées, une série de simulations est effectuée en faisant varier la largeur du modèle 


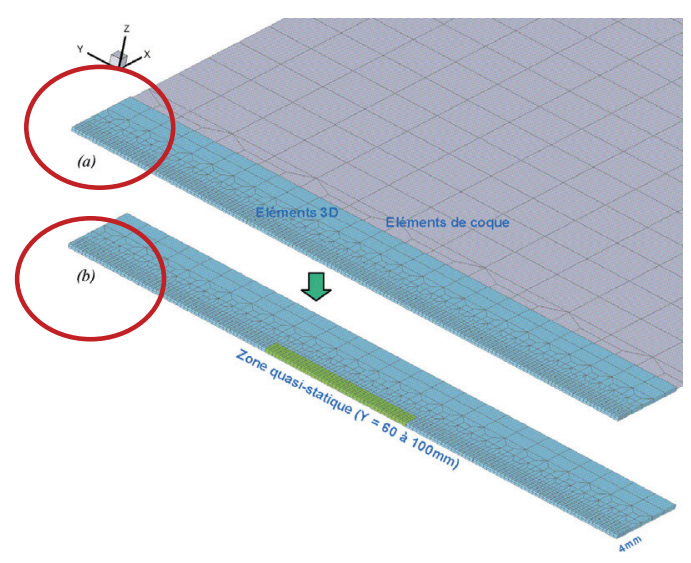

Fig. 3. Modèles locaux utilisés pour optimiser la dimension (a) maillage pour la thermique; b) maillage pour la mécanique).

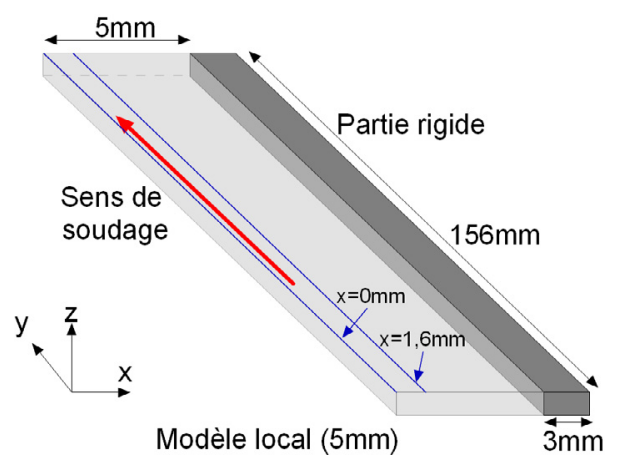

Fig. 4. Conditions rigides appliquées à la frontière en ajoutant une partie rigide (largeur du modèle local $=5 \mathrm{~mm}$ ).

local (partie 3D dans l'analyse thermique) de $5 \mathrm{~mm}$ à $80 \mathrm{~mm}$ et en considérant deux types de conditions aux limites « frontière libre $»$ et « frontière rigide $»$. Ce dernier type de condition consiste à ajouter une partie très rigide à la frontière du modèle local afin de garder cette dernière plane (Fig. 4).

Le modèle local dont la largeur est de $5 \mathrm{~mm}$ et la partie rigide de $3 \mathrm{~mm}$ sont représentés sur la figure 4 . Une valeur du module de Young 3 fois supérieure à celle correspondant au module à froid de l'acier 16MND5 est utilisée dans la partie rigide.

Afin d'effectuer la comparaison entre les résultats des modèles locaux et ceux du modèle de référence, on considère les déformations plastiques dans une petite zone de largeur approximative $4 \mathrm{~mm}$ (Fig. 3). La valeur moyenne de la déformation plastique équivalente de la zone quasi-stationnaire, est considérée pour étudier l'influence des conditions aux limites sur les déformations plastiques calculées. Cette valeur moyenne est rapportée à celle obtenue sur le calcul de référence $\left(S_{1} / S_{\mathrm{r}}\right)$. L'évolution de ce rapport, dans le cas d'une source de chaleur peu pénétrante, est présentée sur la figure 5. Les résultats du modèle local avec « frontière libre » deviennent insensibles à la largeur du modèle local quand cette largeur dépasse $50 \mathrm{~mm}$, avec un écart d'environ $3 \%$. Cet écart est imputé au fait que la contrainte structurelle

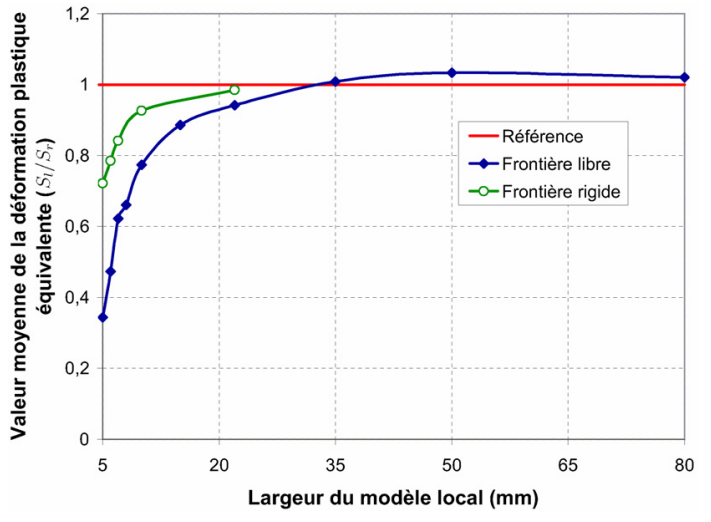

Fig. 5. Valeur moyenne de la déformation plastique équivalente $\left(S_{1} / S_{\mathrm{r}}\right)$ de la zone choisie avec une source de chaleur peu pénétrante.

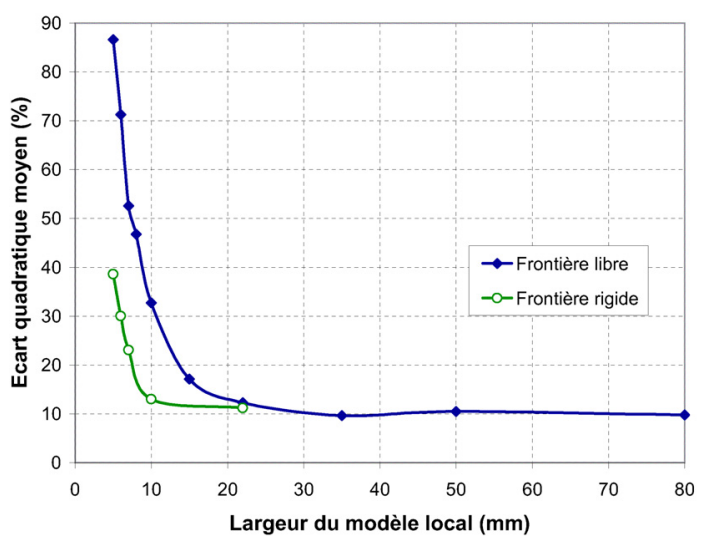

Fig. 6. Écarts quadratiques moyens sur les déformations plastiques équivalentes en fonction de la largeur du modèle local.

du modèle de référence ne peut pas être prise en compte, par les modèles locaux simplifiés de type plaque. Les calculs avec « frontière rigide » conduisent à des résultats toujours meilleurs que ceux avec « frontière libre ».

Les écarts quadratiques moyens sur les déformations plastiques équivalentes par rapport au modèle de référence, concernant les types de conditions aux limites, sont illustrés sur la figure 6 . Ces écarts moyens sont calculés par :

$$
\bar{e}=\frac{\sqrt{\frac{1}{n} \sum_{i=1}^{n}\left(\varepsilon_{i}^{l}-\varepsilon_{i}^{*}\right)^{2}}}{\varepsilon_{\text {moyenne }}^{*}}
$$

où $\varepsilon_{i}^{l}$ désigne la déformation plastique du $i^{\text {ème }}$ nœud du modèle local, $\varepsilon_{i}^{*}$ la déformation plastique du $i$ ème nœud du modèle de référence et $\varepsilon_{\text {moyenne }}^{*}$ la déformation plastique moyenne du calcul de référence.

Comme on peut le constater, pour les calculs avec « frontière libre », les écarts moyens deviennent presque insensibles à la largeur du modèle local quand celle-ci devient supérieure à $22 \mathrm{~mm}$. En outre, les calculs avec 《 frontière libre » donnent des résultats acceptables avec un écart d'environ $10 \%$. Lorsque la largeur est inférieure à $22 \mathrm{~mm}$, les calculs avec « frontière rigide» permettent une importante diminution de l'écart. Cependant, l'écart 
moyen est de $38 \%$ pour le modèle de $5 \mathrm{~mm}$, ce qui reste inacceptable. Par conséquent, il est nécessaire d'augmenter la dimension latérale du modèle local pour obtenir de meilleurs résultats.

\subsection{Influence de la source de chaleur}

Le modèle de référence, utilisé pour une source de chaleur peu pénétrante (Fig. 2), ne peut pas être employé ici. Ceci est dû au fait que dans le cas d'une source de chaleur pénétrante, ce modèle n'est pas assez long pour créer une zone quasi-stationnaire au milieu de la soudure en raison des effets de bord. La longueur de ce modèle est ainsi portée à $276 \mathrm{~mm}$. Le maillage de ce modèle est composé de 35513 nœuds et 12305 éléments quadratiques (6670 éléments de peau, 5635 éléments 3D).

La source de chaleur pénétrante est composée d'une source volumique de $50 \mathrm{~W} . \mathrm{mm}^{-3}$, de forme cylindrique (rayon $=1,5 \mathrm{~mm}$ ), ainsi que d'une source surfacique en forme de gaussienne de rayon $2,5 \mathrm{~mm}$ dont l'intensité maximale est de $15 \mathrm{~W} \cdot \mathrm{mm}^{-2}$. Cette source est déplacée à une vitesse de $10 \mathrm{~mm} . \mathrm{s}^{-1}$ pour laisser un cordon de $260 \mathrm{~mm}$ en $26 \mathrm{~s}$.

À l'aide de la technique de « transformation de l'espace », les distributions de température sont homogènes à partir d'environ $5 \mathrm{~mm}$, comme dans le cas de la source de chaleur peu pénétrante. Il est donc raisonnable de choisir cette valeur pour largeur minimum du maillage local utilisé dans la simulation mécanique.

Deux types de conditions aux limites, « frontière libre » et « frontière rigide » sont considérés dans cette partie. Comme précédemment, les modèles locaux prolongés avec des éléments coques sont utilisés pour les calculs thermo-métallurgiques couplés (Fig. 3), en prenant la largeur de la partie 3D supérieure ou égale à $5 \mathrm{~mm}$.

Bien que le modèle de référence soit prolongé, la longueur des modèles locaux est encore prise égale à $156 \mathrm{~mm}$. La largeur du modèle local (partie 3D dans l'analyse thermique) est prise de $5 \mathrm{~mm}$ à $30 \mathrm{~mm}$ en considérant les deux types de conditions aux limites afin d'étudier la définition du modèle local avec ce type de source de chaleur. Comme dans le cas précédent, pour la condition de type « frontière rigide », une valeur du module de Young $3 E$ est utilisée par la partie rigide.

La figure 7 représente l'évolution de la valeur moyenne de la déformation plastique équivalente dans la zone choisie rapportée à celle du modèle de référence, dans le cas d'une source de chaleur pénétrante. L'écart inhérent du modèle local (lorsque il est assez large), avec les conditions aux limites « frontière libre », est de $8 \%$. Cette valeur est plus grande que celle observée dans le cas de la source de chaleur peu pénétrante $(3 \%)$, et elle est atteinte dès que la largeur du modèle local est supérieure à $22 \mathrm{~mm}$.

De plus, les valeurs moyennes de déformation plastique équivalente s'approchent très rapidement de celle de référence $(12 \mathrm{~mm}$ pour « frontière libre » et $9 \mathrm{~mm}$ pour « frontière rigide »), tandis qu'avec l'option « frontière rigide $»$ et dans le cas de la source de chaleur peu pénétrante

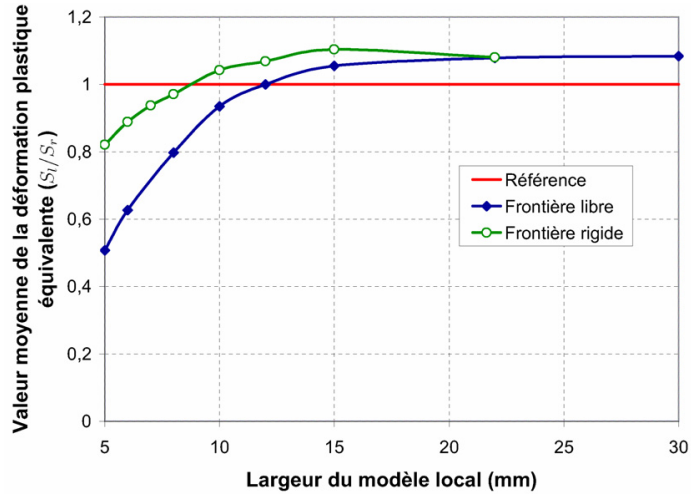

Fig. 7. Valeur moyenne de la déformation plastique équivalente $\left(S_{1} / S_{\mathrm{r}}\right)$ de la zone choisie avec une source de chaleur pénétrante.

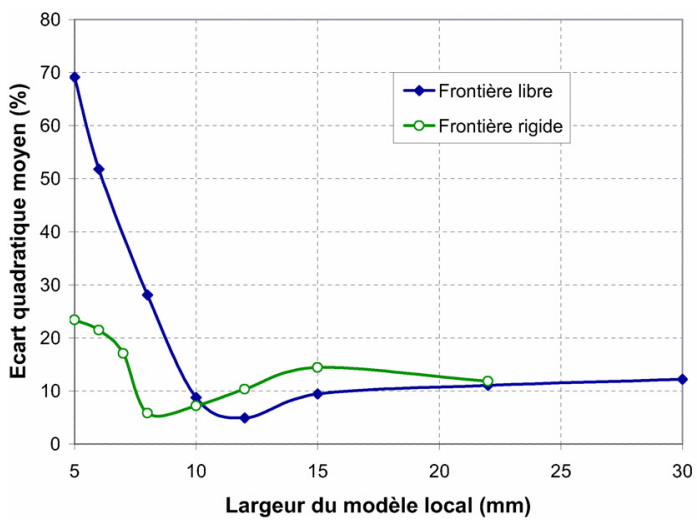

Fig. 8. Écarts quadratiques moyens sur les déformations plastiques équivalentes avec une source de chaleur pénétrante.

ce dépassement ne s'est pas encore produit pour une largeur de $22 \mathrm{~mm}$.

Lorsque la largeur du modèle local est relativement petite, dans le cas $5 \mathrm{~mm}$ par exemple, la valeur moyenne de la déformation plastique équivalente est de 0,50 pour l'option « frontière libre » et de 0,82 pour l'option « frontière rigide $»$. Ces résultats sont meilleurs que dans le cas d'une source de chaleur peu pénétrante.

Les écarts quadratiques moyens sur les déformations plastiques équivalentes concernant les deux types de conditions aux limites, «frontière libre » et « frontière rigide », sont illustrés sur la figure 8 . Comme on peut le constater, les écarts moyens deviennent presque insensibles à la largeur du modèle local lorsque cette dernière devient supérieure à $15 \mathrm{~mm}$. Cependant, les calculs donnent des résultats acceptables avec un écart de l'ordre de $10 \%$ dès que la largeur du modèle local dépasse $10 \mathrm{~mm}$ avec l'option « frontière libre » et $8 \mathrm{~mm}$ avec l'option « frontière rigide ».

\section{Conclusions et perspectives}

La détermination des distorsions résiduelles des structures soudées étant difficile, l'approche locale/globale 
présentée dans cet article semble être une méthode très prometteuse, en particulier pour l'optimisation des séquences de soudage dans un contexte industriel.

Cet article a présenté une démarche originale pour déterminer les dimensions du modèle local. Afin de refléter les conditions de soudage telles qu'elles existent sur la structure réelle, la largeur et les conditions aux limites appliquées à la frontière du modèle local ont été étudiées. Un type de conditions aux limites dit « frontière rigide $»$ qui permet une diminution importante de l'écart moyen a été proposé. Les résultats avec les modèles locaux simplifiés, assez larges et de type plaque, peuvent être utilisés pour calculer les déformations plastiques de grandes structures, néanmoins avec un écart d'environ $3 \%$ sur la valeur moyenne des déformations plastiques équivalentes dans le cas d'une source de chaleur peu pénétrante et de $8 \%$ dans le cas d'une source de chaleur pénétrante.

Finalement, les dimensions du modèle local dépendent aussi de l'épaisseur de la structure; l'influence de ce paramètre devra donc être étudiée. Cette étude pourra être conduite à l'aide du modèle simplifié proposé. Une méthodologie déterminant les dimensions optimales du modèle local pourra alors être proposée.

Remerciements. Ces travaux s'inscrivent dans le programme INZAT 4 et ont été réalisés au laboratoire LTDS en association avec l'Université Paris 6/LMM. Les partenaires industriels de ces travaux sont EADS/CCR, EDF/SEPTEN, ESI-GROUP, FRAMATOME-ANP, avec la participation de la Région Rhône-Alpes.

Toutes les simulations ont été réalisées avec le logiciel SYSWELD ${ }^{\mathrm{TM}}$ [9] développé par ESI-GROUP.

\section{Références}

[1] Ph. Mourgue, Y. Gooroochurn, J.-M. Bergheau, F. Boitout, H. Porzner, P. Niedenzu, Modeling welding sequence on industrial parts, in Proc. of EUROPAM'2000, 10th Int. Conf. and Exhibition on Virtual Prototyping by Numerical Simulation, Nantes, France, 2000., p. 9

[2] M. Näsström, L. Wikander, L. Karlsson, L.-E. Lindgren, J. Goldak, Combined 3D and shell modelling of welding, ed. E. Kozeshnik H. Cerjack, H.K.D.H. Bhadeshia, Mechanical effects of welding - IUTAM Symposium, Lulea, Sweden, 1991, pp. 197-206

[3] Y. Ueda, M. Yuan, Prediction of residual stresses in buttwelded plates using inherent strains, ASME J. Eng. Mat. Tech. 115 (1993) 417-423

[4] P. Michaleris, A. DeBiccari, Prediction of welding distortion, Welding J. 76 (1997) 172-180

[5] P. Duranton, J. Devaux, V. Robin, P. Gilles, J.-M. Bergheau, 3D modelling of multipass welding of a 316L stainless steel pipe, J. Mat. Proc. Tech. 153-154 (2004) 457-463

[6] F. Faure, Nouvelles méthodes pour la simulation numérique du soudage des structures élancées, Ph.D. Thèse, Université de Paris VI, Paris, France, 2004

[7] B. Souloumiac, F. Boitout, J.M. Bergheau, A new local global approach for the modelling of welded steel component distortions, ed. Pr. H. Cerjack, Mathematical Modelling of Weld Phenomena 6, London, 2002, Maney Publishing, pp. 573-590

[8] F. Waeckel, Une loi de comportement thermométallurgique des aciers pour le calcul mécanique des structures, Ph.D. Thèse, École Nationale Supérieure d'Arts et Métiers, France, 1994

[9] SYSTUS/SYSWELD, User's manual, ESI Group, 2006 